\title{
Erratum to: Beta-Blockers and Ivabradine in Chronic Heart Failure: From Clinical Trials to Clinical Practice
}

\author{
Antonino Di Franco • Filippo M. Sarullo • \\ Ylenia Salerno - Stefano Figliozzi - Rossella Parrinello • \\ Pietro Di Pasquale • Gaetano A. Lanza
}

Published online: 24 July 2014

(C) Springer International Publishing Switzerland 2014

Erratum to: Am J Cardiovasc Drugs (2014) 14:101-110

DOI 10.1007/s40256-013-0057-9

Page 106, column 1, paragraph 5, lines 7-8: The following text, which previously read:

NYHA class II-II

Should read:

NYHA class II-III

The online version of the original article can be found under doi:10.1007/s40256-013-0057-9.

\footnotetext{
A. Di Franco - S. Figliozzi - R. Parrinello · G. A. Lanza Department of Cardiovascular Medicine, Università Cattolica del Sacro Cuore, Rome, Italy

F. M. Sarullo $(\square)$ · Y. Salerno

Cardiovascular Rehabilitation Unit, Buccheri La Ferla

Fatebenefratelli Hospital, Via Messina Marine n. 197,

90123 Palermo, Italy

e-mail: fsarullo@neomedia.it

P. Di Pasquale

Division of Cardiology "Paolo Borsellino",

G.F. Ingrassia Hospital, Palermo, Italy
} 